\title{
Assessing the Improvement of Cluster Enteral Nutrition Treatment for Head and Neck Cancer Patients
}

\author{
Laoyan Weng ${ }^{1}$, Yuyao Liu ${ }^{1, ~ *, ~ X i a o f a n g ~ Z h a n g ~}{ }^{1}$, Wenhui Chen ${ }^{1}$, Kaiyin $\mathrm{He}^{2}$, Huatong Wang ${ }^{1}$ \\ ${ }^{1}$ Oncology Department, The First Affiliated Hospital of Jinan University, Guangzhou, China \\ ${ }^{2}$ Nutritional Department, The First Affiliated Hospital of Jinan University, Guangzhou, China
}

\section{Email address:}

531019941@qq.com (Laoyan Weng),787642815@qq.com (Yuyao Liu), hill_zxf@qq.com (Xiaofang Zhang), wenhuichen221@126.com (Wenhui Chen),173234563@qq.com (Kaiyin He),76097681@qq.com (Huatong Wang)

${ }^{*}$ Corresponding author

\section{To cite this article:}

Laoyan Weng, Yuyao Liu, Xiaofang Zhang, Wenhui Chen, Kaiyin He, Huatong Wang. Assessing the Improvement of Cluster Enteral Nutrition Treatment for Head and Neck Cancer Patients. Journal of Cancer Treatment and Research. Vol. 8, No. 1, 2020, pp. 21-24. doi: $10.11648 /$ j.jctr.20200801.14

Received: February 21, 2020; Accepted: March 3, 2020; Published: March 10, 2020

\begin{abstract}
Objective: Assess the improvement of cluster enteral nutrition treatment for head and neck cancer patients. Methods: 104 participants was invested to join our study from December 2016 to December 2018. They were randomly assigned to intervention group and control group in study beginning. For intervention group, we provide additional cluster enteral nutrition treatment to them. In the process, we use the questionnaires, interview and follow-up to collect the data of them, the questionnaires included Scored Patient-Generated Subjective Global Assessment and Nutritional risk screening. The data shows the changing of nutrition status of participants in the process. Result: Base on albumin and transferrin of participants, the intervention had better improvement than that of control group $(1.97 \pm 0.24$ vs $1.61 \pm 0.20,56.72 \pm 2.33$ vs $50.17 \pm 2.01)$. In dietary assessment, intervention group had higher score than control group (11.34 $\pm 1.61 \mathrm{vs} 9.04 \pm 1.35)$. But in nutritional risk assessment, the change gap was not significant in the result. In different complications, the most of complication was nausea and vomiting in the participants $[16(30.77 \%)$ vs $7(13.46 \%)]$. Conclusion: the cluster enteral nutrition treatment had strongly influence for nutrition status of head and neck cancer patients. Moreover, the patients of intervention group had better nutrition status after cluster enteral nutrition treatment. Besides, the cluster enteral nutrition treatment provide better restore to head and neck cancer patients.
\end{abstract}

Keywords: Head and Neck Cancer, Nutrition, Complications

\section{Introduction}

The standard of care in locally advanced head and neck cancer is combined chemoradiation. Even in very advanced stages, the loco-regional control rates are promising with $85 \%$ still alive after a follow-up of five years from chemoradiation [1-3]. In head and neck patients, the nutrition is a serious problem in treatment process. Over $80 \%$ of head and neck cancer patients who have received radiotherapy suffer from radiotherapy-induced xerostomia (dry mouth) [4]. Xerostomia affects cancer patients' quality of life by altering their taste which leads to loss of appetite, interrupting their sleep (due to the frequent need of drinking water) and makes routine activities such as chewing and swallowing a laborious task $[5,6]$.
Head and neck cancer is the 7th most common carcinoma worldwide [7]. Radiotherapy either alone or in combination with surgery or chemotherapy is recommended in the treatment of head and neck cancer $[8,9]$. In some studies, the percentage of malnutrition could even reach as high as $88 \%$ at the end of the treatment [10-12]. So weight loss is an important indicator for nutritional status, is very common in the patients of head and neck cancer, with $43 \%-71 \%$ of the head and neck cancer patients experiencing $>=5 \%$ weight loss during radiotherapy [13-15]. The Aim of our research is assess the improvement of cluster enteral nutrition treatment for head and neck radiotherapy patients. 


\section{Methods}

We invited 104 patients who were diagnosed as head and neck cancer in The First Affiliated Hospital of Jinan University to join our study. Their data was collected and time of follow-up from December 2016 to December 2018. The participants, they all meet our screening criteria, the patients were randomly assigned to two groups which were intervention group $(n=52)$ and a control group $(n=52)$. In the prior-treatment and post-treatment, we record nutritional status [albumin $(\mathrm{g} / \mathrm{L}) \&$ transferrin $(\mathrm{g} / \mathrm{L})]$, weight, physical power and complications on participants. Besides, we assess dietary score and nutritional risk score by Scored Patient-Generated Subjective Global Assessment (PG-SGA), Nutritional risk screening (NRS 2002).

Their inclusion criteria were: (1) the patients received cluster enteral nutrition treatment in radiotherapy treatment process; (2) the result of NRS 2002 assessment was higher than 3 scores; (3) the tumor did not metastasize; (4) no major organ failure or serious dysfunction. Their withdraw criteria were: (1) the patients had hepatic and renal failure; (2) they had gastrointestinal complications. In addition, all participants voluntarily participated in this study and signed informed consent.

\section{Result}

We collected the data of albumin and transferrin with head and neck cancer patients. Base on Table 1, there was significant change from nursing before to after nursing. Following to change, the intervention had better improvement than that of control group $(1.97 \pm 0.24$ vs $1.61 \pm 0.20$, $56.72 \pm 2.33$ vs $50.17 \pm 2.01)$. Besides, the part of improvement was statistical significance $(\mathrm{p}<0.005)$.

Table 1. Nutritional Status [albumin $(g / L) \&$ transferrin $(g / L)]$.

\begin{tabular}{|c|c|c|c|c|c|c|c|c|}
\hline \multirow{2}{*}{ Projects } & \multicolumn{4}{|c|}{ Transferrin (g/L) (Mean \pm SD) } & \multicolumn{4}{|c|}{$\operatorname{Albumin}(\mathrm{g} / \mathrm{L})($ Mean \pm SD) } \\
\hline & BN & FN & T-text & P Value & BN & FN & T-text & P Value \\
\hline Control Group $(\mathrm{n}=52)$ & $2.15 \pm 0.18$ & $1.61 \pm 0.20$ & 14.472 & $<0.005$ & $60.24 \pm 5.07$ & $50.17 \pm 2.01$ & 13.314 & 0.024 \\
\hline Intervention Group $(\mathrm{n}=52)$ & $2.13 \pm 0.19$ & $1.97 \pm 0.24$ & 3.770 & $<0.005$ & $60.11 \pm 4.83$ & $56.72 \pm 2.33$ & 4.558 & $<0.005$ \\
\hline T-text & 0.551 & 8.309 & - & - & 0.134 & 15.349 & - & - \\
\hline P Value & 0.583 & 0.041 & - & - & 0.894 & $<0.005$ & - & - \\
\hline
\end{tabular}

$\mathrm{BN}=$ before the nursing.

$\mathrm{FN}=$ after the nursing.

We assess the dietary and nutritional risk of patients by PG-SGA questionnaire and NRS 2002 questionnaire. Both of control group and intervention group had slightly improvement in dietary health and nutritional risk (Table 2). In dietary assessment, intervention group had higher score than control group (11.34 $\pm 1.61 \mathrm{vs} 9.04 \pm 1.35)$. But in nutritional risk assessment, the change gap was not significant in the result.

Table 2. The Result of Dietary Assessment and Nutritional Risk Assessment.

\begin{tabular}{|c|c|c|c|c|c|c|c|c|}
\hline \multirow{2}{*}{ Projects } & \multicolumn{4}{|c|}{ Dietary Assessment (Mean \pm SD) } & \multicolumn{4}{|c|}{ Nutritional Risk Assessment (Mean \pm SD) } \\
\hline & BN & $\mathbf{F N}$ & T-text & P Value & BN & FN & T-text & P Value \\
\hline Control Group $(\mathrm{n}=52)$ & $8.15 \pm 1.29$ & $9.04 \pm 1.35$ & 3.437 & $<0.005$ & $4.78 \pm 1.96$ & $6.98 \pm 2.45$ & 5.056 & $<0.005$ \\
\hline Intervention Group $(\mathrm{n}=52)$ & $8.22 \pm 1.47$ & $11.34 \pm 1.61$ & 10.320 & $<0.005$ & $4.72 \pm 2.04$ & $5.26 \pm 2.10$ & 1.330 & 0.186 \\
\hline T-text & 0.258 & 7.894 & - & - & 0.153 & 3.844 & - & - \\
\hline P Value & 0.797 & 0.031 & - & - & 0.879 & $<0.005$ & - & - \\
\hline
\end{tabular}

$\mathrm{BN}=$ before the nursing.

$\mathrm{FN}=$ after the nursing.

The information of weight and physical power was collected from hospital database. In weight, the patients of control group loss more weight, but it means the patients of control group had worse nutritional status (Table 3). On the contrary, the patients of intervention group had better score of physical power assessment, it shown the patients of intervention group had better nutritional status.

Table 3. Weight and Physical Power Assessment.

\begin{tabular}{|c|c|c|c|c|c|c|c|c|}
\hline \multirow{2}{*}{ Projects } & \multicolumn{4}{|c|}{ Weight (KG) (Mean \pm SD) } & \multicolumn{4}{|c|}{ Physical Power Assessment (Mean \pm SD) } \\
\hline & BN & FN & T-text & P Value & BN & FN & T-text & P Value \\
\hline Control Group $(\mathrm{n}=52)$ & $68.22 \pm 2.70$ & $57.24 \pm 3.12$ & 19.190 & $<0.005$ & $42.14 \pm 6.09$ & $35.52 \pm 8.06$ & 4.726 & $<0.005$ \\
\hline Intervention Group $(\mathrm{n}=52)$ & $68.24 \pm 2.68$ & $66.87 \pm 2.15$ & 2.875 & $<0.005$ & $42.17 \pm 6.13$ & $37.93 \pm 7.47$ & 3.164 & 0.022 \\
\hline T-text & 0.038 & 18.327 & - & - & 0.025 & 1.581 & - & - \\
\hline P Value & 0.970 & $<0.005$ & - & - & 0.980 & 0.117 & - & - \\
\hline
\end{tabular}

$\mathrm{BN}=$ before the nursing.

$\mathrm{FN}=$ after the nursing.

In complications status, patients of intervention group had less complications after the treatment (Table 4). In different 
complications, the most of complication was nausea and vomiting in the participants [16 (30.77\%) vs 7 (13.46\%)]. Additionally, the oral ulcer data was statistical significance $(\mathrm{p}=0.012)$.

Table 4. Complications Status.

\begin{tabular}{llll}
\hline Projects & Oral Ulcer & Loss of Appetite or Dry Mouth & Nausea and Vomiting \\
\hline Control Group $(\mathrm{n}=52)$ & $12(23.08 \%)$ & $7(13.46 \%)$ & $16(30.77 \%)$ \\
Intervention Group $(\mathrm{n}=52)$ & $3(5.77 \%)$ & $5(9.62 \%)$ & $7(13.46 \%)$ \\
$X^{2}$ & 6.310 & 0.377 & 4.522 \\
P Value & 0.012 & 0.539 & 0.033 \\
\hline
\end{tabular}

\section{Discussion}

In $2018,2.6 \%$ of new cancer diagnosis and $2.7 \%$ of cancer related deaths in the world were caused by head and neck cancer [16]. Base on the report, people with head and neck cancer (HNC) report many disease and health-related problems before, during and a long time after completion of their treatment [17].

Base on the outcome of this study, the cluster enteral nutrition treatment had strongly influence for nutrition status of head and neck cancer patients. Following to albumin and transferrin of participants, they shown the change was associated with nutrition status, the patients of intervention group had better nutrition status after cluster enteral nutrition treatment. Similarly, the improvement was associated with the weight, physical power, dietary health and nutritional risk of participants was present that the cluster enteral nutrition treatment provides better restore to head and neck cancer patients. Finally, number of complications was reduced after cluster enteral nutrition treatment, even through the improvement was not significant, it had not strongly influence for the complications of patients.

\section{Conclusion}

In conclusion, the cluster enteral nutrition treatment improves the outcome of head and neck cancer patients in the treatment process. Its improvement included 4 domains with head and neck cancer patients, such as nutrition status; the dietary and nutritional risk; weight and physical power; complications status. Moreover, the cluster enteral nutrition treatment had great influence for nutritional status, the nutritional status of intervention group had significant improvement in the result. However, the result of complications status was rare, a part of data was not statistical significant, because the simple size was limit the information of complications status.

\section{References}

[1] Gillison ML et al. Radiotherapy plus cetuximab or cisplatin in human papillomavirus-positive oropharyngeal cancer (NRG Oncology RTOG 1016): a randomised, multicentre, non-inferiority trial. Lancet 2019; 393 (10166): 40-50.

[2] Dorth JA, Patel PR, Broadwater G, et al. Incidence and risk factors of significant carotid artery stenosis in asymptomatic survivors of head and neck cancer after radiotherapy. Head
Neck 2014; 36: 215-219.

[3] Carpenter DJ, Mowery YM, Broadwater G, et al. The risk of carotid artery stenosis in head and neck cancer patients after radiation therapy. Oral Oncol 2018; 80: 9-15.

[4] Pinna R, Campus G, Cumbo E, Mura I, Milia E, Xerostomia induced by radiotherapy: an overview of the physiopathology, clinical evidence, and management of the oral damage, Ther. Clin. Risk Manag. 2015: 171-188.

[5] Jensen SB, Pedersen AM, Vissink A, Andersen E, Brown CG, Davies AN, et al., A systematic review of salivary gland hypofunction and xerostomia induced by cancer therapies: prevalence, severity and impact on quality of life, Support Care Cancer. 2016; 10 (8): 1039-1060.

[6] Hatakeyama $\mathrm{H}$ et al. Osteoradionecrosis of the hyoid bone after intra-arterial chemoradiotherapy for oropharyngeal cancer: MR imaging findings. Cancer Imaging 2017; 17 (1): 22.

[7] Torre LA, Bray F, Siegel RL, Ferlay J, Lortet-Tieulent J, Jemal A. Global cancer statistics. CA Cancer J Clin. 2015; 65: 87108 .

[8] Galbiatti AL, Padovani-Junior JA, Maníglia JV, Rodrigues CD, Pavarino ÉC, Goloni-Bertollo EM. Head and neck cancer: causes, prevention and treatment. Braz J Otorhinolaryngol. 2013; 79: 239-247.

[9] Raguse JD, Hossamo J, Tinhofer I, et al. Patient and treatment-related risk factors for osteoradionecrosis of the jaw in patients with head and neck cancer. Oral Surg Oral Med Oral Pathol Oral Radiol Endod 2016; 121 (3): 215-21.

[10] Unsal D, Mentes B, Akmansu M, Uner A, Oguz M, Pak Y. Evaluation of nutritional status in cancer patients receiving radiotherapy. Am. J. Clin. Oncol. 2016; 29: 183-188.

[11] Mahdavi R, Faramarzi E, Mohammad-Zadeh M, Ghaeammaghami J, Jabbari MV. Consequences of radiotherapy on nutritional status, dietary intake, serum zinc and copper levels in patients with gastrointestinal tract and head and neck cancer. Saudi Med. J. 2017; 28: 435-440.

[12] Platek AJ, Jayaprakash V, Merzianu M, et al. Smoking cessation is associated with improved survival in oropharynx cancer treated by chemoradiation. Laryngoscope. 2016; 126 (12): 2733-2738.

[13] Kubrak C, Olson K, Jha N, Scrimger R, Parliament M, McCargar L, Koski S, Baracos VE. Clinical determinants of weight loss in patients receiving radiation and chemoirradiation for head and neck cancer: a prospective longitudinal view. Head Neck. 2013; 35: 695-703.

[14] Lonbro S, Petersen GB, Andersen JR, Johansen J. Prediction of critical weight loss during radiation treatment in head and neck cancer patients is dependent on BMI. Support. Care Cancer. 2016; 24: 2101-2109. 
[15] Jager-Wittenaar H, Dijkstra PU, Vissink A, van der Laan BF, van Oort RP, Roodenburg JL. Critical weight loss in head and neck cancer-prevalence and risk factors at diagnosis: an explorative study. Support. Care Cancer. 2017; 15: 1045-1050.

[16] Bray F, Ferlay J, Soerjomataram I, Siegel RL, Torre LA, Jemal A, Global cancer statistics 2018: GLOBOCAN estimates of incidence and mortality worldwide for 36 cancers in 185 countries, CA Cancer J. Clin. 2018; 68 (6): 394-424.
[17] Rogers SN, Lowe D, Lowies C, Yeo ST, Allmark C, Mcavery D, Humphris GM, Robert Flavel R, Semple C, Thomas SJ, Kanatas A. Improving quality of life through the routine use of the patient concerns inventory for head and neck cancer patients: a cluster preference randomized controlled trial. BMC Canc. 2018; 18: 444. 